\title{
Texture analysis on MR images helps predicting non-response to NAC in breast cancer

\author{
N. Michoux ${ }^{*}$, S. Van den Broeck ${ }^{2}$, L. Lacoste ${ }^{2}$, L. Fellah ${ }^{2}$, C. Galant ${ }^{3}$, M. Berlière ${ }^{4}$ and I. Leconte ${ }^{2}$
}

\begin{abstract}
Background: To assess the performance of a predictive model of non-response to neoadjuvant chemotherapy (NAC) in patients with breast cancer based on texture, kinetic, and BI-RADS parameters measured from dynamic MRI.

Methods: Sixty-nine patients with invasive ductal carcinoma of the breast who underwent pre-treatment MRI were studied. Morphological parameters and biological markers were measured. Pathological complete response was defined as the absence of invasive and in situ cancer in breast and nodes. Pathological non-responders, partial and complete responders were identified. Dynamic imaging was performed at 1.5 T with a 3D axial T1W GRE fat-suppressed sequence. Visual texture, kinetic and BI-RADS parameters were measured in each lesion. ROC analysis and leave-one-out cross-validation were used to assess the performance of individual parameters, then the performance of multi-parametric models in predicting non-response to NAC.
\end{abstract}

Results: A model based on four pre-NAC parameters (inverse difference moment, GLN, LRHGE, wash-in) and k-means clustering as statistical classifier identified non-responders with $84 \%$ sensitivity. BI-RADS mass/non-mass enhancement, biological markers and histological grade did not contribute significantly to the prediction.

Conclusion: Pre-NAC texture and kinetic parameters help predicting non-benefit to NAC. Further testing including larger groups of patients with different tumor subtypes is needed to improve the generalization properties and validate the performance of the predictive model.

Keywords: Breast cancer, Neoadjuvant chemotherapy, MRI, Texture analysis

\section{Background}

Neoadjuvant chemotherapy (NAC) has a major role in the treatment of breast cancer $[1,2]$. Several trials comparing adjuvant chemotherapy and NAC demonstrated that long-term relapse-free and overall survival outcomes were the same [3]. However, NAC has advantages compared with adjuvant chemotherapy. NAC can safely downstage tumor so that conservative surgery can be performed instead of mastectomy [4, 5]. Importantly, NAC is the only way to study the effect of systemic chemotherapy in vivo and to identify prognostic factors. However, the rate of response to NAC is limited and dependent on the subtypes of cancer [6-12]. It has been recently reported that pathological complete response

\footnotetext{
*Correspondence: nicolas.michoux@uclouvain.be

'Radiology Department, IREC (Institute of Experimental and Clinical Research) IMAG, Université Catholique de Louvain, Cliniques Universitaires Saint-Luc, Avenue Hippocrate 10, Brussels B1200, Belgium

Full list of author information is available at the end of the article
}

(pCR) obtained after NAC is a suitable surrogate endpoint for disease-free survival in patients with luminal B/Human Epidermal growth factor Receptor 2 (HER2) -negative, HER2-positive (non-luminal) and triple negative tumors but not for those with luminal B/HER2-positive or luminal A tumors. However, the rate of $\mathrm{pCR}$ in these different breast cancer subtypes varies from 6 to $33 \%$ [13]. Therefore, the identification of non-responding patients is important, especially as it may allow considering alternative therapeutic options.

The predictive value of Magnetic Resonance Imaging (MRI) and in particular of diffusion-weighted MRI [14-16], MR spectroscopy [17-19] or Dynamic Contrast-Enhanced MRI (DCE-MRI) [20-23] has been investigated. However, most of these studies were performed after the first courses of NAC. Some studies reported that certain pre-NAC semi-quantitative DCE parameters were significantly different in chemosensitive and chemoresistant breast lesions 
and may contribute to the prediction of disease-free survival and overall survival [24-26].

Alternative quantitative approaches such as visual texture analysis have been considered [27, 28]. Texture analysis allows for the description of the MR appearance of the tissues and of their changes in terms of fineness, coarseness, smoothness, granularity, homogeneity or periodicity [29]. These attributes are related to the local spatial distribution of the grey levels in the image matrix and can be captured by using metrics, called texture parameters. In texture analysis of MR images, it is assumed that the distribution of the grey levels results from the underlying ultrastructural properties of tissues affected by the disease processes-an assumption that has been validated by finding correlation between MRI texture patterns and tissue changes on histological analysis [30]. Numerically, texture can be described by using $\mathrm{n}^{\text {th }}$-order statistics, spatial frequency or structural primitives, the first two approaches being the most commonly used. A practical description of the concepts and methodologies for texture analysis of MR images has been proposed by Hajek et al. [31]. First studies in breast MRI, while remaining to be validated, showed that certain pretreatment texture parameters (based on high order statistics) may help evaluate breast tumor response to NAC [32-34].

The aim of the study is to assess the value of preNAC imaging parameters to predict non-responders to NAC. To this purpose, texture, kinetic and BI-RADS (Breast Imaging-Reporting and Data System) parameters were studied from baseline MRI. Thence, a three-step assessment was undertaken. First, texture parameters were compared in healthy breast tissues and in tumor lesions. Secondly, the performance of individual parameters in predicting pathological non-response to NAC was assessed. Thirdly, parameters were combined into multi-parametric models. The predictive performance of these multi-parametric models was then assessed after cross-validation.

\section{Methods}

\section{Patients}

This two-years retrospective study was approved by our institutional ethical committee (Comité d'Ethique hospitalo-facultaire, Cliniques Universitaires Saint-Luc, http://www.comite-ethique-ucl-saintluc.be/). Written informed consent from the patients was not required. All patients had an invasive breast carcinoma diagnosed on core-biopsy specimen. To obtain a homogeneous histological sample for texture analysis, only invasive ductal carcinomas (IDC) with and without ductal carcinoma in situ (DCIS) were included in this pilot study. The mean number of cancers-newly diagnosed in our institution was 296 per year. Seventeen percent of patients with invasive cancers received NAC. The percentage of in situ (DCIS and LCIS) was comprised between 17 to $21 \%$.

A baseline MRI as well as a pre-operative MRI to evaluate response to NAC was performed in all patients. After multidisciplinary breast cancer tumor board decision, all patients underwent NAC, consisting of 4 cycles of cyclophosphamide/anthracyclines followed by 4 cycles of taxanes [2,3] and trastuzumab in case of HER2+ tumor. Cycles were administrated every 3 weeks. All patients had surgery three to four weeks after completing NAC. As a result, the delay between diagnosis and surgery was approximately 6 months.

Patients with incomplete pathological and radiological data $(n=6)$ and severe artifacts on MRI images (e.g. respiratory motion and body movement) $(n=3)$ were excluded. Overall, this retrospective study included 69 patients with IDC (median age 54 years, range 22-72 years). Estrogen receptor (ER), progesterone receptor (PgR) and, HER2 status as well as the mitotic factor Ki67 were available on percutaneous biopsies. Patients' characteristics are listed in Table 1.

\section{Pathological and biological analysis}

Breast tissues sampled for histopathological analysis were sectioned at the macroscopic level transversally in

Table 1 Patients characteristics $(n=69)$. Number and proportions within the whole population are given

\begin{tabular}{|c|c|}
\hline Characteristics & Values \\
\hline Median age (range) & $54(22-72)$ \\
\hline \multicolumn{2}{|l|}{ BI-RADS feature } \\
\hline Mass & $39(57 \%)$ \\
\hline non mass & $30(43 \%)$ \\
\hline \multicolumn{2}{|l|}{ Histological grade } \\
\hline IDC 1 & 0 \\
\hline $\operatorname{IDC} 2$ & $25(36 \%)$ \\
\hline IDC 3 & $44(64 \%)$ \\
\hline \multicolumn{2}{|l|}{ Subtypes } \\
\hline Luminal A & $13(19 \%)$ \\
\hline Luminal B/HER2- & $25(36 \%)$ \\
\hline Luminal B/HER2+ & $15(22 \%)$ \\
\hline Non luminal/HER2+ & $10(14 \%)$ \\
\hline Triple-negative & $6(9 \%)$ \\
\hline \multicolumn{2}{|l|}{ Receptor status } \\
\hline ER positivity & $52(75 \%)$ \\
\hline PgR positivity & $42(61 \%)$ \\
\hline Ki67 > $14 \%$ & $52(75 \%)$ \\
\hline HER2 positivity & $26(38 \%)$ \\
\hline Triple-negative cancer rate & $6(9 \%)$ \\
\hline
\end{tabular}

$I D C$ invasive ductal carcinoma, $E R$ estrogen receptor, $P g R$ progesterone receptor, HER2 epidermal growth factor receptor 2 
order to produce $10 \mathrm{~mm}$ slices. A dedicated breast pathologist analyzed each lesion at the microscopic level, describing first the size of every residual cancerous foci and then classifying these into three categories according to the NSABP 18 criteria [35]: pathological complete (CR), partial (PR) and non-response (NR). In case of a single mass lesion with a concentric response, the size of the residual tumor was measured. In case of a single masse lesion with a fragmented response, i) the overall dimension of the foci is given when foci are adjacent, ii) each foci is measured when foci are distant and the overall sum is given. In case of a non-mass lesion with fragmented response, the overall size is given.

The density of tumor cells, compared to the previous biopsy, was also analyzed, allowing the classification of the tumor following the grading system of Miller-Payne (5 grades). The tumor grade was evaluated with the Nottingham score.

A pathological complete response was defined as the absence of invasive and in situ cancer in breast and nodes. A partial response was defined as a decrease of invasive cancer exceeding $30 \%$. A non-response was defined as a decrease of invasive cancer lower than $30 \%$. At histological analysis, 14 patients were thus classified as CR, 36 as PR and 19 as NR.
All biological markers were evaluated on percutaneous biopsies. As regards immunohistochemical assessments, IDCs were classified according to their receptor status. ER and PgR were considered as negative when $<10 \%$ nuclei stained positive [36]. For all lesions, the results for HER 2 expression by immunohistochemical analysis were scored as $0,1+, 2+$ and $3+$. Only $3+$ specimens were immediately considered as HER2-positive. A hybridization technique was performed when analysis score was $2+$. Both negative and $1+$ were considered as negative. The mitotic activity marker Ki67 was considered as positive when expressed by more than $14 \%$ of tumor cells [13]. Correlation between sensitivity of breast cancer to NAC and receptor status is given in Table 2 .

\section{MRI sequence}

MRI examinations were performed using a $1.5 \mathrm{~T}$ whole body imaging system (Gyroscan Intera, Philips Medical System, The Netherlands) and a breast coil. Patients were imaged in the prone position with T2-weighted and diffusion-weighted imaging (DWI) (b0, b600) sequences, and a 3D gradient echo axial T1-weighted sequence with fat suppression (SPAIR). Scan parameters were TR/TE $=4.8$ / $2.4 \mathrm{~ms}$, flip angle $=10^{\circ}, \mathrm{FOV}=355 \times 355 \mathrm{~mm}$, matrix $320 \times$ 320 , slice thickness $2.5 \mathrm{~mm}$, voxel size $0.65 \times 0.65 \times 1.25 \mathrm{~mm}$

Table 2 Association between pathologic responsiveness of breast cancer to NAC and receptor status

\begin{tabular}{|c|c|c|c|c|c|}
\hline Pathologic response & NR & $C R$ & PR & $\mathrm{PR}+\mathrm{CR}$ & $p$-value ${ }^{a}$ \\
\hline \multicolumn{6}{|l|}{ BI-RADS } \\
\hline Mass & $12(31 \%)$ & & & $27(69 \%)$ & 0.51 \\
\hline non Mass & 7 (23\%) & & & $23(77 \%)$ & 0.51 \\
\hline \multicolumn{6}{|l|}{ Biological markers } \\
\hline ER positivity & $15(29 \%)$ & 9 (17\%) & $28(54 \%)$ & 37 (71 \%) & 0.70 \\
\hline PgR positivity & $14(33 \%)$ & $4(10 \%)$ & $24(57 \%)$ & $28(67 \%)$ & 0.19 \\
\hline Ki67> $14 \%$ & $11(21 \%)$ & $11(21 \%)$ & $30(58 \%)$ & 41 (79 \%) & 0.05 \\
\hline HER2 positivity & $4(15 \%)$ & 8 (31%) & $14(54 \%)$ & $22(85 \%)$ & 0.09 \\
\hline \multicolumn{6}{|l|}{ Subtypes } \\
\hline Luminal A & 8 (62 \%) & 0 & 5 (38 \%) & 5 (38 \%) & 0.005 \\
\hline Luminal B/ HER2 - & 4 (16\%) & $5(20 \%)$ & 16 (64 \%) & $21(84 \%)$ & 0.11 \\
\hline Luminal B/HER2 + & $3(20 \%)$ & 4 (27 \%) & 8 (53 \%) & $12(80 \%)$ & 0.49 \\
\hline Non-luminal/HER2 + & 1 (10 \%) & $4(40 \%)$ & $5(50 \%)$ & 9 (90 \%) & 0.20 \\
\hline Triple-negative cancer rate & $3(50 \%)$ & 1 (17\%) & 2 (33\%) & $3(50 \%)$ & 0.25 \\
\hline \multicolumn{6}{|l|}{ Histological grade } \\
\hline IDC 2 & $5(20 \%)$ & $3(12 \%)$ & 17 (68 \%) & $20(80 \%)$ & 0.31 \\
\hline IDC 3 & $14(32 \%)$ & $11(25 \%)$ & 19 (43\%) & $30(68 \%)$ & 0.31 \\
\hline
\end{tabular}

The number and proportions of NR, CR, PR and PR + CR patients with a given feature within all patients having this feature are given. The statistical significance of the relationship between response ( $N R$ or $P R+C R$ ) and features is then assessed ( $p$-value ${ }^{\mathrm{a}}$ ). If a $p$-value $<0.05$ is observed for a given feature, then we can conclude that patients' response is associated to that feature. If a $p$-value $>0.05$ is observed, then the null hypothesis that there is no association, cannot be rejected. Subtype Luminal $A$ is the only feature showing a significant association with response

$B I-R A D S$ breast imaging-reporting and data system, NR non response, $C R$ complete response, $P R$ partial response, $E R$ estrogen receptor, $P g R$ progesterone receptor, Ki67 cellular marker for proliferation based on monoclonal antibody Ki-67, HER2 human epidermal growth factor receptor $2, H R$ hormone receptor, IDC invasive ductal carcinoma

${ }^{\text {a }}$ Significance of the association between response (NR or PR $+C R$ ) and features (Fisher's exact test) 
after reconstruction. The anatomic study was followed by a dynamic study. Patients received $0.1 \mathrm{mmol} / \mathrm{kg}$ of gadobenate dimeglumine (Multihance, Bracco Imaging, Germany) followed by $30 \mathrm{~mL}$ saline flush injected at a rate of $2 \mathrm{~mL} / \mathrm{s}$ with an automated injector. One pre- and five post-injection images were acquired with a temporal resolution of approximately $60 \mathrm{~s}$. The total acquisition time for the protocol was about $6 \mathrm{~min}$. Analyses were performed on subtracted images, i.e. the residual difference image obtained after the second post-contrast image has been subtracted from the pre-contrast image.

\section{Image analysis}

Magnetic resonance images in 69 patients were reviewed consensually by a trainee and two experienced radiologists (10 and 15 years of breast MRI experience respectively) without knowledge of the pathological findings or mammographic and sonographic data, by using the
American College of Radiology BI-RADS MR lexicon [37]. Lesions were categorized into mass enhancement and non-mass enhancement (Fig. 1 and Table 2). The uni- or multifocal character of the lesion was reported. In case of multifocal lesion, only the findings of the largest lesion were recorded. The slice exhibiting the largest dimension of the lesion on the second post-contrast image (enhancement peak) was chosen for analysis. This criterion was applied in case of mass enhancement or non-mass enhancement.

For kinetic analysis, a small region of interest (ROI) corresponding to the most enhancing area of the lesion was drawn (Fig. 2). The size of the ROI always included more than nine pixels [38]. According to the BI-RADS guidelines, characteristics of the signal intensity $v s$ time curve (i.e. the maximal amplitude, the wash-in and the delayed phase pattern via the wash-out parameter) were assessed.
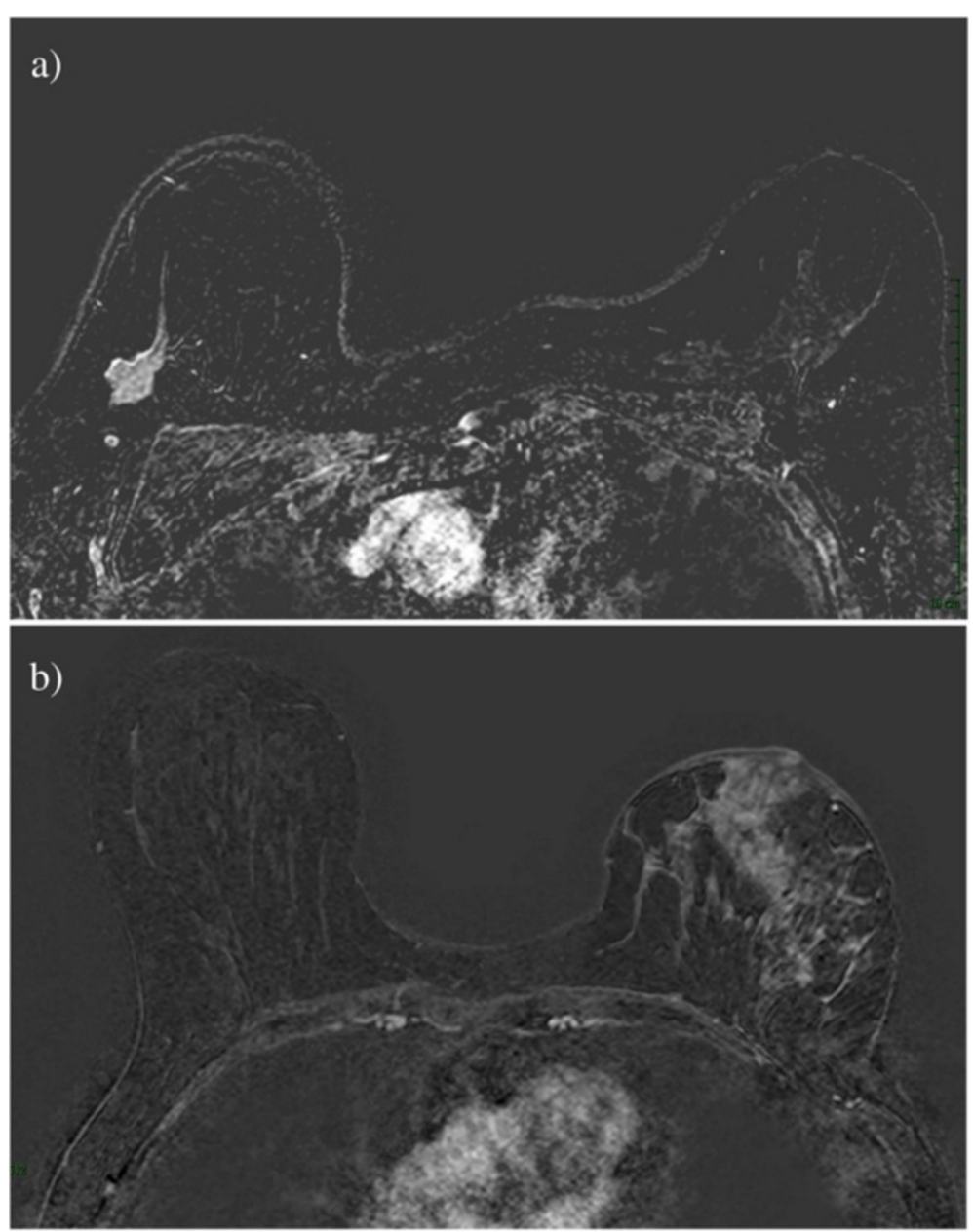

Fig. 1 Axial subtracted images. According to the BI-RADS MR lexicon, the tumor is described as, a ovalar mass with spiculated margins and a homogenous enhancement in the upper external quadrant, or $\mathbf{b}$ retro-areolar non mass lesion, showing a cobblestone-like pattern with nipple invasion and skin thickening 
For texture analysis, a first ROI delimiting healthy tissues was drawn. Healthy tissues were delimited in a remote area of the lesion in the same breast, or in the contralateral breast in case of very large lesions. Based on texture differences observed between fat and healthy tissues (data not shown), healthy tissues were defined as fibroglandular tissues excluding fatty tissues. This distinction was always feasible as none of the patients studied had exclusively fat breast. A second ROI delimiting the lesion was drawn. The lesion was defined as the largest area with a high enhancement, excluding macro vessels. As this definition may be operator dependent, an automated segmentation was also implemented (Fig. 3). In brief, a rectangular ROI was defined in order to cover the whole breast. For each pixel within this ROI, parameters amplitude and wash-in were calculated. A $k$-means clustering algorithm was used to partition the pixels into 2 clusters (lesion and non-lesion) [39]. Then, a morphological opening was applied to remove isolated groups of pixels. The result of the segmentation was the largest region of contiguous pixels with the same behavior in amplitude and wash-in. This result was validated by comparison with the ROI drawn manually.

The visual texture of breast tissues was assessed from the grey level co-occurrence matrix (GLCM) and the run length matrix (RLM) $[29,40]$. From the GLCM, nine textural features describing the grey levels interdependence in the image were estimated (Fig. 4). Computation parameters were: distance of one pixel between two neighbouring pixels, average of the angular relationships on the four main directions, five bits of grey levels. From the RLM, eleven textural features describing the distribution of runs of grey levels in the image were estimated with the same computation parameters. The mean value (over all pixels in the ROI studied) of the textural features was estimated. The list of studied parameters is given in Table 3.

\section{Statistical analysis}

Numerical variables are expressed as median and range (95\% CI on the median). The three-step comparative approach was conducted as follows. First, texture parameters were compared in healthy breast tissues $v s$

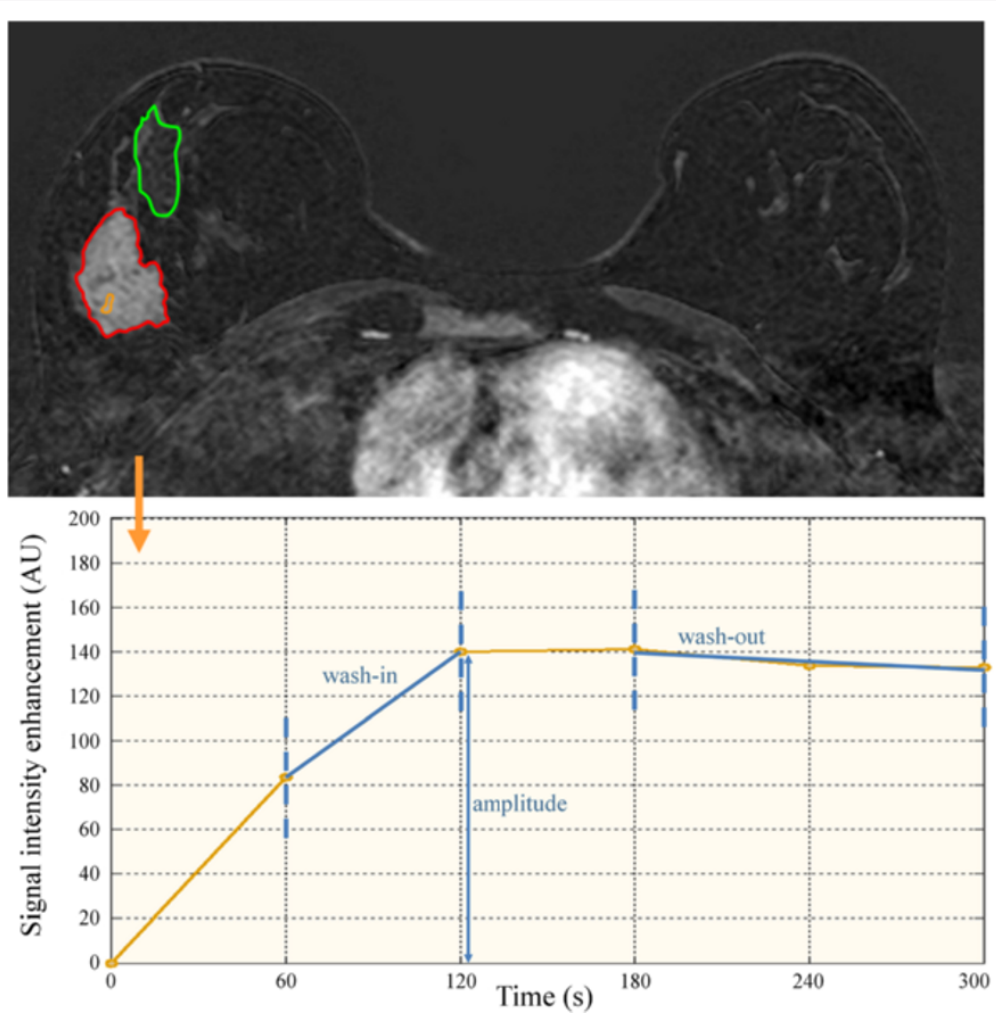

Fig. 2 Top, axial fat-suppressed T1 weighted imaging (time corresponding to the second post-contrast image). Two large ROls, one encompassing the lesion (in red) and one encompassing normal breast tissues (in green), were defined for visual texture analysis. A small ROI (in yellow) in the brightest part of the lesion was also defined to study the kinetics of the contrast agent. Bottom, the signal intensity vs time curve (temporal sampling 60 s) corresponding to the small $\mathrm{ROI}$ (from which kinetic parameters are derived) is displayed. Amplitude was calculated from the maximum enhancement peak, the wash-in parameter from the up-slope measurement (between the maximum enhancement peak and the preceding time point) and the wash-out parameter from linear regression performed on the last three time points of the signal intensity versus time curve 

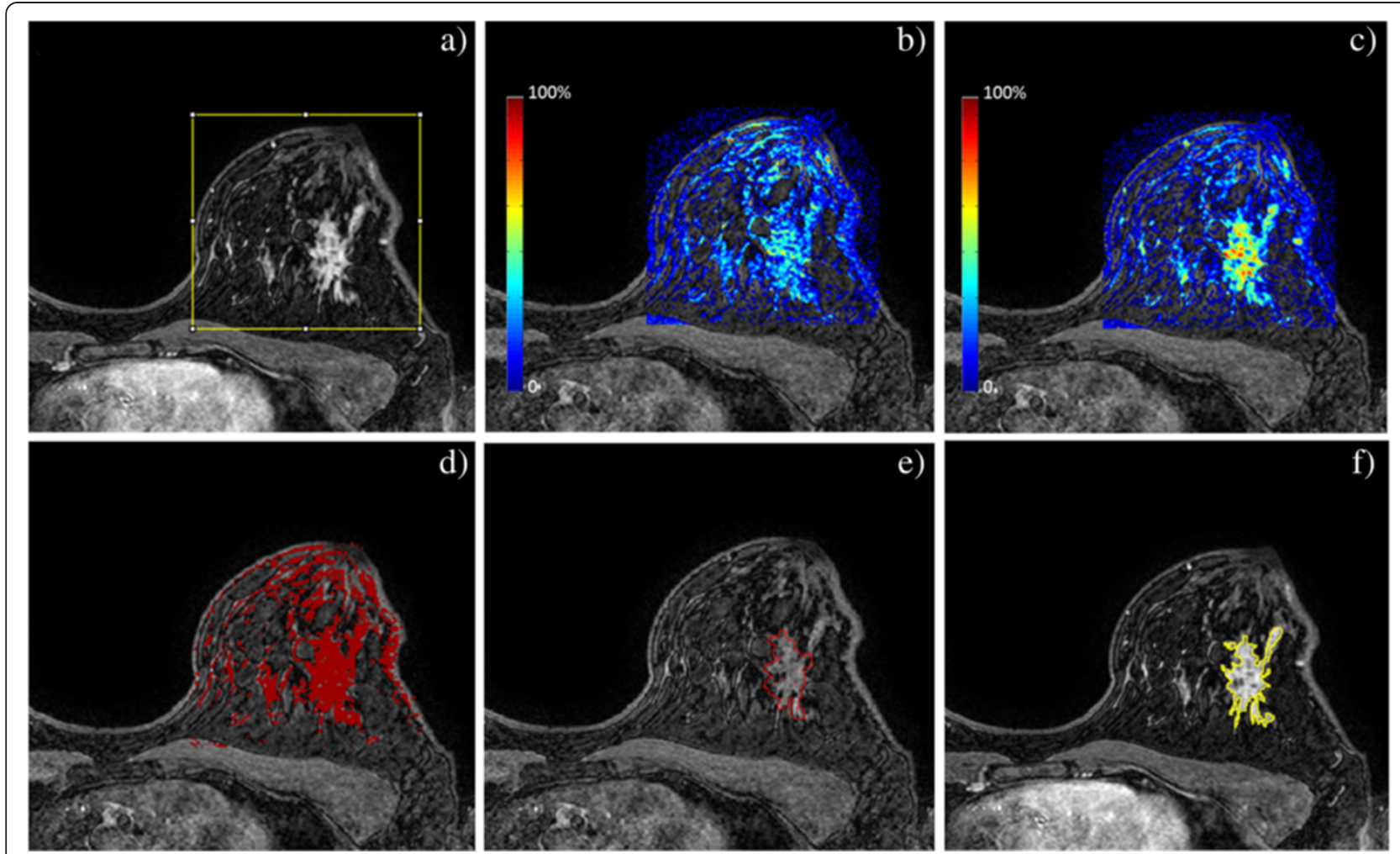

Fig. 3 Automated segmentation of the tumor lesion. A rectangular area covering the breast is placed (a). Pixel-level calculation of parameters wash-in (b) and amplitude (c) is performed. Pixels are partitioned into $k=2$ clusters (d). Morphological opening is applied to preserve the largest region of contiguous pixels with the same behavior in amplitude and wash-in only (e). Comparison with the manual delineation of the lesion shows an overall good agreement (f)

tissues showing characteristics of a malignant lesion. A Wilcoxon rank-sum test was performed. This nonparametric test was chosen as the normality of the data distribution was not verified (on the basis of the D’Agostino-Pearson test).

Secondly, texture, kinetic, BI-RADS and biological parameters were compared in $\mathrm{NR} v s \mathrm{PR}+\mathrm{CR}$ individually. A mid-P approach of Fisher's exact test was performed for assessing the relationship between response (NR or $\mathrm{PR}+\mathrm{CR}$ ) and features [41]. The performance of each parameter in predicting non-response to NAC was assessed by using receiver operating characteristic (ROC) curves and by comparing Area Under the ROC Curves (AUC) [42].

Thirdly, texture, kinetic, BI-RADS and biological parameters were combined. Two multi-parametric classifiers, each belonging to one of the two classes of algorithms in machine learning (supervised and unsupervised), were tested: a logistic regression model [43] and a $k$-means clustering algorithm based on a nearest-cluster approach [39]. The $k$-means algorithm was parameterized with a number of final clusters equal to 2, 2 random observations to choose the initial cluster centroid positions, 30 replicates and with the L1 distance to calculate the distance between centroid clusters. As one cannot know a priori how many and which parameters are important to the classification, all possible combinations of 2 to 26 parameters among 26 parameters (20 texture parameters, 3 kinetic parameters, the mass/non-mass enhancement, Ki67 > 14 \%, HR/HER2) were submitted to the classifiers successively.

To estimate how accurately the predictive models would perform in practice, a leave-one-out cross validation was applied [44]. The cross validation works by leaving one observation (i.e. one patient data) out each time the classifier is trained. Thus, the observation can be used to test the classifier accuracy. The operation is then carried out for all observations. Hence, the percentage of NR patients classified correctly (i.e. the classifier sensitivity, Se) and the percentage of $\mathrm{PR}+\mathrm{CR}$ patients classified correctly (i.e. the classifier specificity, Sp) were estimated. Se and Sp were finally used to identify the set of features that yielded best predictive models.

All calculations (texture computation and statistics) were done with Matlab (Matlab R2011b, MathWorks, Natick, MA, USA). Open source codes "KeyRes-Technologies" 

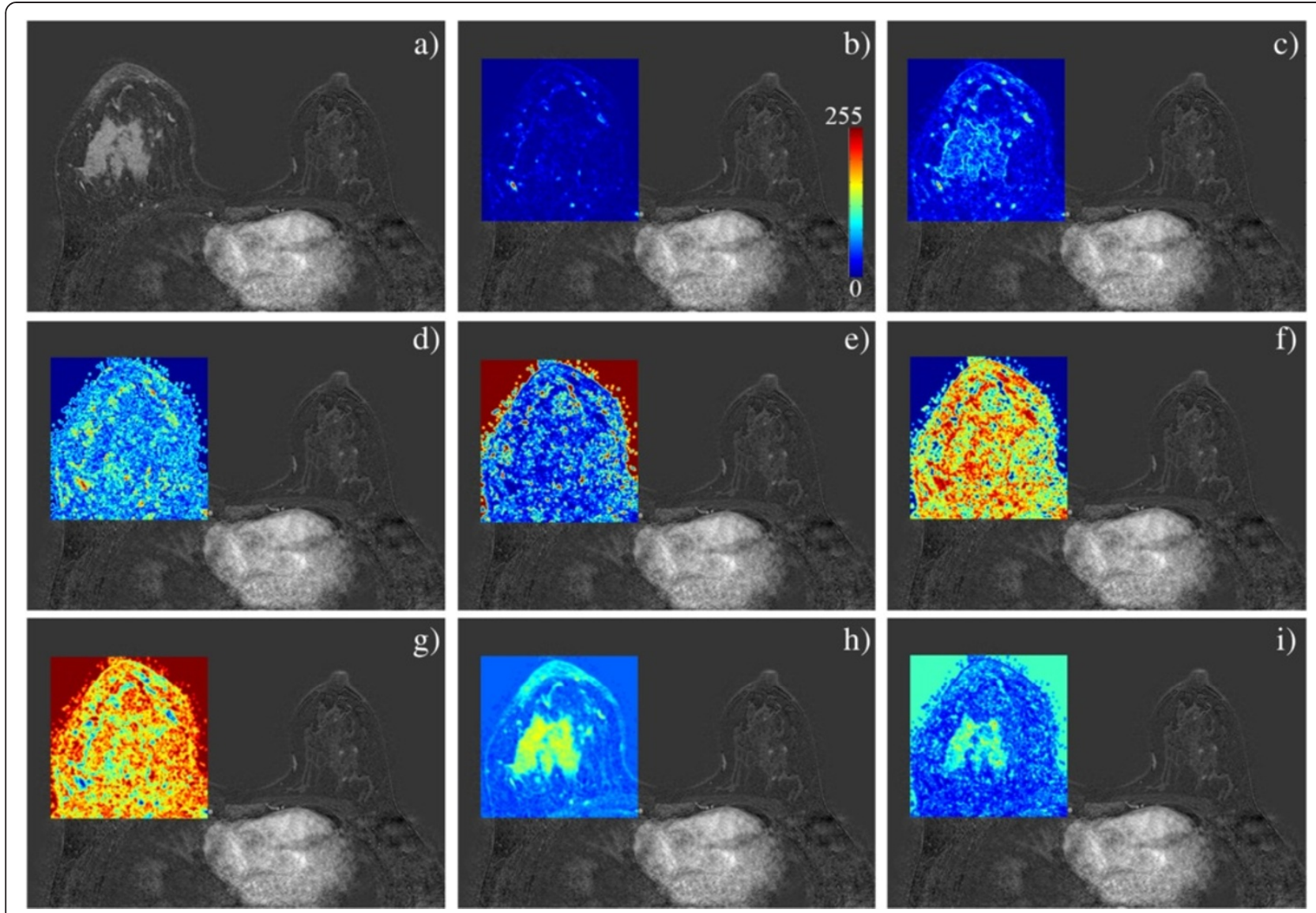

Fig. 4 Pixel-level analysis of breast MRI texture in a CR patient with a mass enhancement. Are respectively displayed, a the axial subtracted image and the maps based on $\mathbf{b}$ contrast, c correlation, $\mathbf{d}$ difference variance, e energy, $\mathbf{f}$ entropy, $\mathbf{g}$ inverse differential moment (which is correlated with the homogeneity parameter), $\mathbf{h}$ sum average and $\mathbf{i}$ sum variance from the GLCM, with mean value estimated on a $3 \times 3$ neighbourhood around the pixel of interest then normalized on the 0-255 range. Individual texture parameters reveal different local and regional statistical properties of the grey level intensity between (and respectively within) breast lesions and normal parenchyma. Combination of all or parts of the texture parameters helps classifying patients according to their response to NAC

and "grayrlmatrix" under Matlab were used for computing texture parameters. The software Image J (http://rsbweb.nih.gov/ij/) was used for the segmentation of the ROIs. A $p$-value $<0.05$ was considered as statistically significant for all tests cited above, as the universal null hypothesis was of no interest in this study [45].

\section{Results}

\section{Biological and imaging parameters}

Morphological, biological and histological findings are reported in Table 2. Neither the mass enhancement nor the non-mass enhancement were statistically different between NR and PR + CR. NR were significantly more represented in Luminal-A subtype compared to PR + CR. NR were significantly less represented in Ki67 > 14 \% and HR-/HER2+ compared to PR + CR (non-significant trend). No statistical difference on histological grade between NR and PR + CR was observed.
Texture and kinetic parameters are reported in Table 4. Significant differences between healthy tissues and malignant tissues were observed for all texture parameters (all $p$-value $<0.05$ ).

\section{Mono-parametric prediction}

AUC values, sensitivity and specificity of selected cut-offs are given for all parameters in Table 5. Parameters energy, entropy, homogeneity inverse difference moment, RP, HGRE and wash-in were found to have an AUC significantly different from $0.5\left(^{\text {energy }}=0.002, p^{\text {entropy }}=0.003\right.$, $p^{\text {homogeneity }}=0.001, p^{\text {inv. diff. mom. }}=0.001, p^{\text {diff. var. }}=0.023$, $\left.p^{\mathrm{RP}}=0.045, p^{\mathrm{HGRE}}=0.038, p^{\text {wash-in }}=0.008\right)$. The performance associated with these parameters ranged from fair $(0.5<\mathrm{AUC} \leq 0.7)$ to $\operatorname{good}(0.7<\mathrm{AUC} \leq 0.9)$. The pairwise comparison of AUCs did not allow ranking strictly these parameters according to their individual performance ( $p>0.05$ whatever the comparison). 
Table 3 List of parameters used for breast lesion characterization

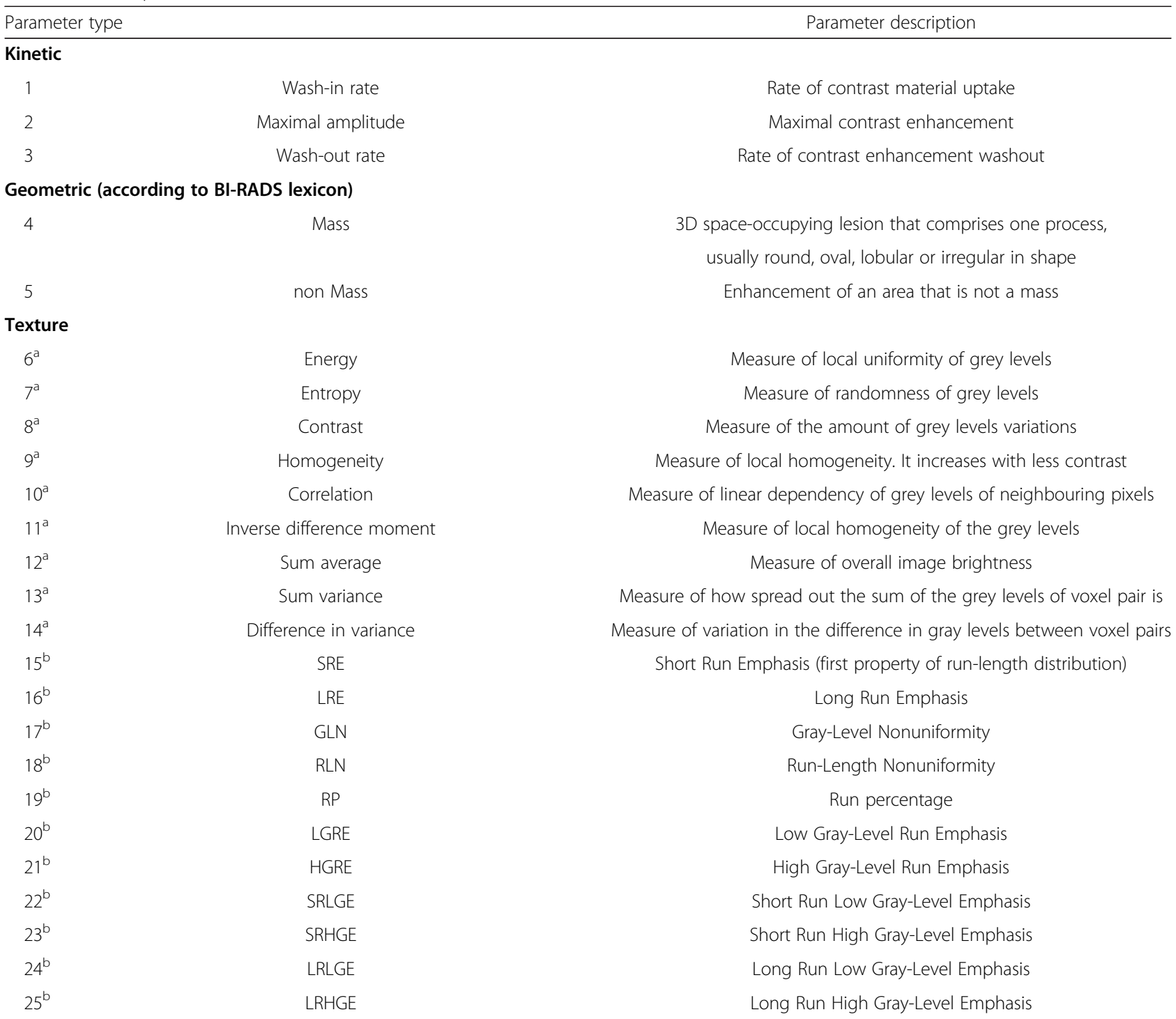

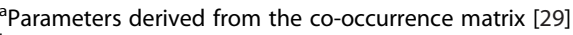

${ }^{\mathrm{b}}$ Parameters derived from the run length matrix [40]

$3 D$ three-dimensional, BI-RADS breast imaging reports and data system

\section{Multi-parametric prediction}

Using $k$-means clustering as classifier, a predictive model relying on four parameters (inverse difference moment, GLN, LRHGE, wash-in) was found to perform with a predictive accuracy of $68 \%: \mathrm{Se}=84 \%(16 / 19 \mathrm{NR})$ and $\mathrm{Sp}=62 \%(31 / 50 \mathrm{PR}+\mathrm{CR})$. Using log-transformed parameters (energy, homogeneity, wash-in, LRHGE), it was possible to increase the percentage of NR classified correctly up to $95 \%$ (18/19), but with a lower specificity of $32 \%(16 / 50 \mathrm{PR}+\mathrm{CR})$ and a lower predictive accuracy of $64 \%$. Using logistic regression as classifier, a more parsimonious predictive model was found. It was based on two texture parameters only (homogeneity, LGRE) and exhibited a predictive accuracy of $74 \%$ : Se $=74 \%(14 / 19$ $\mathrm{NR})$ and $\mathrm{Sp}=74 \%(37 / 50 \mathrm{PR}+\mathrm{CR})$. Models using other combinations and/or a larger number of parameters did not improve the predictive accuracy (regardless of the type of classifier).

\section{Discussion}

The first observation of this study is that texture analysis discriminates healthy breast tissues from tumor lesion. Texture is more heterogeneous and coarse in the enhancing part of the lesion compared to healthy tissue. This observation agrees with previously published results on the ability of visual texture parameters to 
Table 4 Median values (95\% Cl) of the texture and kinetic parameters

\begin{tabular}{|c|c|c|c|c|}
\hline & Normal tissue & $C R+P R$ & NR & $p$-value \\
\hline Energy & $58[44 ; 74]$ & $36[33 ; 41]$ & $45[42 ; 55]$ & $7.910^{-5}$ \\
\hline Entropy & $157[141 ; 172]$ & $187[181 ; 193]$ & $175[165 ; 180]$ & $6.410^{-5}$ \\
\hline Contrast & $8[6 ; 10]$ & $14[11 ; 16]$ & $13[10 ; 16]$ & $8.610^{-5}$ \\
\hline Homogeneity & $165[150 ; 176]$ & $140[134 ; 146]$ & $149[144 ; 156]$ & $5.110^{-5}$ \\
\hline Correlation & $22[18 ; 29]$ & $47[42 ; 52]$ & $47[44 ; 50]$ & $1.810^{-14}$ \\
\hline Inv. Diff. Moment & $174[161 ; 185]$ & $148[141 ; 153]$ & $158[153 ; 165]$ & $4.210^{-5}$ \\
\hline Sum average & $69[65 ; 76]$ & $119[114 ; 124]$ & $120[109 ; 127]$ & $3.610^{-19}$ \\
\hline Sum variance & $70[60 ; 76]$ & $92[88 ; 99]$ & $97[86 ; 110]$ & $2.210^{-15}$ \\
\hline Difference variance & $74[67 ; 81]$ & $87[82 ; 93]$ & $80[78 ; 83]$ & $4.610^{-3}$ \\
\hline SRE & $0.009[0.008 ; 0.009]$ & $0.004[0.0039 ; 0.0044]$ & $0.0038[0.0035 ; 0.0047]$ & $6.210^{-19}$ \\
\hline LRE & $126[114 ; 144]$ & $266[246 ; 279]$ & $284[229 ; 309]$ & $7.610^{-20}$ \\
\hline GLN & $158[137 ; 229]$ & $432[338 ; 589]$ & $416[298 ; 817]$ & $1.210^{-10}$ \\
\hline RLN & $71[58 ; 86]$ & $111[74 ; 120]$ & $105[89 ; 205]$ & $6.210^{-4}$ \\
\hline $\mathrm{RP}$ & $0.68[0.62 ; 0.72]$ & $0.72[0.71 ; 0.75]$ & $0.70[0.66 ; 0.73]$ & $9.310^{-4}$ \\
\hline LGRE & $0.75[0.71 ; 0.78]$ & $0.79[0.78 ; 0.81]$ & $0.77[0.74 ; 0.80]$ & $9.810^{-4}$ \\
\hline HGRE & $3.11[2.54 ; 3.99]$ & $2.55[2.33 ; 2.76]$ & $2.83[2.49 ; 3.34]$ & $8.810^{-3}$ \\
\hline SRLGE & $0.0060[0.0056 ; 0.0067]$ & $0.0033[0.0031 ; 0.0034]$ & $0.0030[0.0028 ; 0.0036]$ & $2.610^{-17}$ \\
\hline SRHGE & $0.028[0.024 ; 0.034]$ & $0.011[0.010 ; 0.012]$ & $0.011[0.009 ; 0.014]$ & $6.410^{-18}$ \\
\hline LRLGE & $93[84 ; 101]$ & $204[189 ; 215]$ & $214[177 ; 251]$ & $6.010^{-20}$ \\
\hline LRHGE & $412[343 ; 509]$ & $679[615 ; 745]$ & 799 [592; 925] & $1.910^{-9}$ \\
\hline Amplitude & - & $75[70 ; 80]$ & $68[59 ; 79]$ & - \\
\hline Wash-out & - & $0.04[0.03 ; 0.06]$ & 0.04 [0.008; 0.070] & - \\
\hline Wash-in & _ & $0.72[0.64 ; 0.83]$ & $0.63[0.42 ; 0.70]$ & _- \\
\hline
\end{tabular}

Amplitude is given in arbitrary unit (AU), wash-in and wash-out in AU. $\mathrm{s}^{-1}$ $N R$ Non response, $C R$ Complete response, $P R$ Partial response

${ }^{a}$ Statistical difference (Wilcoxon) between normal tissues and tumoral lesion

differentiate normal from malignant tissue with breast DCE-MRI [27].

The second observation is that the predictive performance of individual texture and kinetic parameters did not exceed the level fair, except for parameters homogeneity and inverse difference moment whose performance level is evaluated as good.

The third observation is that a multi-parametric model based on texture and kinetic parameters was able to predict non-response to NAC with a good performance level. This observation needs to be discussed according to the study design.

The usefulness of pre-NAC DCE parameters in predicting response to NAC was proven in several studies, however on the basis of different assumptions. While Uematsu et al. [24] suggest that a washout enhancement pattern is related to a more effective cycle of NAC, Pickles et al. [25] conclude that high values of perfusion and capillary permeability indicate a high level of angiogenesis and, are therefore indicative of treatment failure. In our study, a faster contrast agent uptake by the tumor as well as a (non-significant) trend towards a higher washout value were observed in $\mathrm{PR}+\mathrm{CR}$. The increased pre-NAC vascularity and permeability characteristics may be interpretable in terms of better delivery of chemotherapeutic agents to the tumor and better treatment efficacy. However, we think that the assumption of vascular characteristics associated with NAC efficacy must be considered with caution. First, drug resistance is a multifactorial phenomenon where cellular mechanisms have a predominant role [46]. Secondly, standard protocol in dynamic breast MRI based on a high spatial resolution such as the one we used in this study does not meet all requirements for an accurate analysis of transport mechanisms in lesions [47]. Such analysis requires a sampling rate less than the mean transit time of the contrast agent, the measurement of an individual arterial input function, the knowledge of the relationship between signal intensity and contrast agent concentration in the tissues and a pertinent mass transport model [48-50].

The usefulness of pre-NAC texture parameters in predicting response to NAC was confirmed in this study, but based on a partially different set of parameters compared to previously published studies. In [33], an increased 
Table 5 Performance of the individual parameters measured from ROC curves (based on the Youden index for determining cut-offs)

\begin{tabular}{|c|c|c|c|c|}
\hline & Se (\%) & $\mathrm{Sp}(\%)$ & AUC & Cut-offs \\
\hline Energy $^{a}$ & 64 & 79 & 0.702 & 41 \\
\hline Entropy $^{a}$ & 64 & 79 & 0.696 & 182 \\
\hline Contrast & 30 & 95 & 0.576 & 17 \\
\hline Homogeneity $^{a}$ & 58 & 84 & 0.701 & 144 \\
\hline Correlation & 62 & 16 & 0.512 & 42 \\
\hline Inv. Diff. Moment ${ }^{a}$ & 60 & 84 & 0.711 & 152 \\
\hline Sum average & 28 & 90 & 0.527 & 103 \\
\hline Sum variance & 78 & 42 & 0.583 & 104 \\
\hline Difference variance ${ }^{a}$ & 60 & 79 & 0.649 & 86 \\
\hline SRE & 80 & 42 & 0.569 & 0.004 \\
\hline LRE & 86 & 37 & 0.569 & 301 \\
\hline GLN & 74 & 42 & 0.555 & 621 \\
\hline RLN & 38 & 90 & 0.579 & 75 \\
\hline $\mathrm{RP}^{\mathrm{a}}$ & 42 & 90 & 0.640 & 0.740 \\
\hline LGRE & 42 & 90 & 0.630 & 0.800 \\
\hline $\mathrm{HGRE}^{\mathrm{a}}$ & 42 & 90 & 0.644 & 2.40 \\
\hline SRLGE & 70 & 53 & 0.582 & 0.003 \\
\hline SRHGE & 16 & 100 & 0.510 & 0.007 \\
\hline LRLGE & 80 & 37 & 0.536 & 233 \\
\hline LRHGE & 72 & 58 & 0.620 & 781 \\
\hline Amplitude & 67 & 58 & 0.567 & 69.1 \\
\hline Wash-out & 27 & 95 & 0.594 & 0.09 \\
\hline Wash-in ${ }^{a}$ & 86 & 47 & 0.685 & 0.50 \\
\hline Mass $^{b}$ & 63 & 46 & 0.546 & - \\
\hline non Mass ${ }^{b}$ & 63 & 46 & 0.546 & - \\
\hline Ki67 > $14 \%^{\mathrm{b}}$ & 42 & 82 & 0.621 & - \\
\hline HER $2+{ }^{b}$ & 79 & 44 & 0.615 & - \\
\hline $\mathrm{HR}-/ \mathrm{HER} 2+^{\mathrm{b}}$ & 100 & 20 & 0.600 & - \\
\hline
\end{tabular}

An overall better performance of GLCM compared to RLM parameters, as well as a better performance of texture and kinetic parameters compared to BI-RADS and biological parameters was observed

${ }^{\text {a }}$ Parameters performing significantly better than a random classifier $\left.\left(p^{(A \cup C}>0.5\right)<0.05\right)$

${ }^{\mathrm{b} C a t e g o r i a l}$ variables without cut-offs

heterogeneity of the texture indicated by the higher values of two parameters (contrast, difference variance) was observed in NR. However, texture was evaluated from the whole lesion including central necrosis, thus increasing the heterogeneity measurements. In the present study, a reduced heterogeneity of the texture (as indicated by the four significant GLCM parameters) in the enhancing part of the lesion was found in NR compared to PR $+C R$. One of these parameters (inverse difference moment) was found to be predictive of a reduced chemotherapeutic response, but jointly with two RLM parameters (GLN,
LRHGE) whose high values indicate a more heterogeneous distribution of some grey level run lengths in NR. There is no obvious explanation at the histological level for these differences of behavior. Further investigations on how and which texture parameters are associated with tumor biology may help defining on the relationship between texture heterogeneity and response to NAC.

Methodological differences in the assessment of texture limit the comparisons between studies. The most common texture analysis techniques are derived either from grey level histogram [51], gradient matrix [52], GLCM [29], RLM [40], local binary patterns [52], autoregressive model [53], Riesz transform [54], multiple frequency scales [55], S-transform [56] or from wavelet [57]. None of these approaches is superior to the others since their effectiveness basically relies on the visual properties of images to which they are applied. Combining various texture methods may improve the characterization of breast lesions as demonstrated by our data. However, increasing the number of texture parameters has some drawbacks. Dimensionality reduction techniques prior to classification, sophisticated machine learning classifiers as well as larger training datasets become necessary. Our four-parameter predictive model may thus present a practical advantage over those proposed in $[33,34]$ for further testing.

The usefulness of BI-RADS mass/non-mass enhancement could not be validated possibly due to a high prevalence of non-mass lesions in our cohort [8, 24]. Rates of complete responders are known to be different within tumor subtypes [7]. We assumed that the low statistical power induced by the small number of patients within each subtype prevents from observing such difference. Ki67 > 14\% and HR-/HER2+ were the only markers more often seen in responders. These parameters, having a fair performance, were not retrieved in the best predictive model.

The performance of our predictive model, albeit good, appeared lower compared to the one reported in previous studies. In [26, 32, 34], predictive accuracy was 85 , 83 and $88 \%$ respectively. However, comparison is flawed as cross-validation was not performed in either of these studies, while it is necessary to get an unbiased estimate of the predictive accuracy [58]. The use of techniques such as cross-validation, bootstrapping or Bayesian confidence interval should be generalized to get a reliable assessment of classifier performance, useful to estimate the relevance of the working hypothesis and mandatory for clinical acceptance.

Clinical response definition and chemotherapy regimen may influence the predictive accuracy. In [32], the difference between 'good' and 'bad' responders was arbitrarily fixed at $50 \%$ decrease in tumor volume between baseline MRI and after 2 cycles of chemotherapy. We on the other 
hand used the pathological response, which is the gold standard in the assessment of response to NAC. In [34], the predictive accuracy of the model depended on the type of chemotherapy regimen undergone by the patients. A similar report was made by Richard et al. studying the predictive value of pre-treatment apparent diffusion coefficients [59]. This raises the question of whether a generalized predictive model of response to NAC independent of chemotherapy regimen can be established.

There are several limitations to the study. First, this is a retrospective study based on a limited number of patients. While our first dataset served for model learning, a second and larger dataset is necessary to validate the performance of the predictive model. This approach, replicating the most interesting results of the pilot study, will address significance problem that may arise when dealing with a large set of parameters. Besides, various types of machine learning classifier can be envisaged, influencing the performance as well [60]. Further tests may be needed to compare the efficacy and practicality of these classifiers. In this pilot study, a single subtracted MR image was evaluated at a specific time-point corresponding to the enhancement peak on intensity time curves. Subtracted images were chosen because of the attenuation of the normal parenchymal background enhancement. Tests on late time points (i.e. on the fifth and sixth dynamics corresponding to imaging of tumor permeability) did not allow for the identification of a good predictive model. Due to its complexity, multi-slice evaluation based on 3D segmentation of the lesion and 3D texture analysis was not envisaged in first instance. However, 3D is one of the strategies to be considered for improving the prediction of response to NAC. Only patients with invasive ductal carcinoma were included. The choice of a single subtype of cancer, far from constituting a selection bias, is legitimate within a dichotomous approach of the problem of predicting response to NAC. Our outcome score depended on histopathological findings and we wanted therefore to obtain a histologically homogeneous group to test texture analysis. Furthermore, it has been demonstrated that invasive lobular carcinoma is less sensitive to NAC [61]. Other studies emphasized that in ILC, immediate treatment with endocrine therapy might be more beneficial [62]. Finally, though combining texture and kinetic parameters with BI-RADS and biological markers did not presently improve the predictive accuracy, these latter parameters should not be discarded in another framework where different (or several) subtypes of breast cancer would be studied.

\section{Conclusion}

Pre-NAC texture and kinetic parameters measured from dynamic breast MRI help predict non-response of invasive ductal carcinoma to neoadjuvant chemotherapy.
Due to the numerous steps necessary to the processing of DCE-MR images, further investigations are needed. It is especially important to test other texture features and statistical classifiers to improve the overall performance of the model, and to include larger groups of tumor subtypes in order to improve the generalization properties of the predictive model. The rationale behind these investigations is the development of a computer-assisted prediction solution dedicated to breast MRI. Such a solution would be cost-effective in comparison to genetic/molecular assessments and may contribute to an appropriate treatment outcome for patients with breast cancer initially eligible for NAC.

\section{Competing interest}

The authors declare that they have no competing interests.

\section{Authors' contributions}

NM conceived the study, carried out the image processing and the statistical analysis, and drafted the manuscript. SVdB and LL performed the acquisition of breast MR images and drew the regions of interest. LF carried out the patient data management and supervised with IL the radiological interpretation of MR images. CG performed the histological analysis. MB provided the expertise in oncology. IL participated in the design of the study and helped to draft the manuscript. All authors read and approved the final manuscript.

\section{Acknowledgements}

We thank Professor François Duhoux (IREC - Université Catholique de Louvain, Belgium) for his expertise in oncology, and Alain Guillet (SMCS Université Catholique de Louvain, Belgium) for his expertise in data mining.

\section{Author details}

'Radiology Department, IREC (Institute of Experimental and Clinical Research) IMAG, Université Catholique de Louvain, Cliniques Universitaires Saint-Luc, Avenue Hippocrate 10, Brussels B1200, Belgium. ${ }^{2}$ Radiology Department, Cliniques Universitaires Saint-Luc, Avenue Hippocrate 10, Brussels B1200, Belgium. ${ }^{3}$ Surgery Department, Cliniques Universitaires Saint-Luc, Avenue Hippocrate 10, Brussels B1200, Belgium. ${ }^{4}$ Pathology Department, Cliniques Universitaires Saint-Luc, Avenue Hippocrate 10, Brussels B1200, Belgium.

Received: 7 March 2014 Accepted: 16 July 2015

Published online: 05 August 2015

\section{References}

1. Kaufmann M, von Minckwitz G, Smith R, Valero V, Gianni L, Eiermann W, et al. International expert panel on the use of primary (preoperative) systemic treatment of operable breast cancer: review and recommendations. J Clin Oncol. 2003;21:2600-8.

2. Heys SD, Hutcheon AW, Sarkar TK, Ogston KN, Miller ID, Payne S, et al. Neoadjuvant docetaxel in breast cancer: 3-year survival results from the Aberdeen trial. Clin Breast Cancer. 2002;3:S69-74.

3. Van der Hage JA, van de Velde CJ, Julien JP, Tubiana-Hulin M, VanderveldenC DL. Preoperative chemotherapyin primary operable breast cancer: results from the European Organization for Research and Treatment of Cancer trial 10902. J Clin Oncol. 2001;19:4224-32.

4. Mieog JS, van der Hage JA, van de Velde CJ. Preoperative chemotherapy for women with operable breast cancer. Cochrane Database Syst Rev. 2007;18:CD005002.

5. Fisher B, Bryant J, Wolmark N, Mamounas E, Brown A, Fisher ER, et al. Effect of preoperative chemotherapy on the outcome of women with operable breast cancer. J Clin Oncol. 1998;16:2672-85.

6. Barbi GP, Marroni P, Bruzzi P, Nicolò G, Paganuzzi M, Ferrara GB. Correlation between steroid hormone receptors and prognostic factors in human breast cancer. Oncology. 1987;44:265-9.

7. von Minckwitz G, Sinn HP, Raab G. Clinical response after two cycles compared to HER2, Ki-67, p53, and bcl-2 in independently predicting a 
pathological complete response after preoperative chemotherapy in patients with operable carcinoma of the breast. Breast Cancer Res. 2008;10:R30

8. Esserman LJ, Kaplan E, Partridge S, Tripathy D, Rugo H, Park J, et al. MRI phenotype is associated with response to doxorubicin and cyclophosphamide neoadjuvant chemotherapy in Stage III breast cancer. Ann Surg Oncol. 2001;8:549-59.

9. Nishimura R, Osako T, Okumura Y, Hayashi M, Arima N. Clinica significanceof Ki-67 in neoadjuvant chemotherapy for primary breast cancer as a predictor for chemosensitivity and for prognosis. Breast Cancer. 2010;17:269-75

10. Fangberget A, Nilsen LB, Hole KH, Holmen MM, Engebraaten $\mathrm{O}$, Naume B, et al. Neoadjuvant chemotherapy in breast cancer-response evaluation and prediction of response to treatment using dynamic contrast-enhanced and diffusion-weighted MR imaging. Eur Radiol. 2011;21:1188-99.

11. Press MF, Sauter G, Buyse M, Bernstein L, Guzman R, Santiago A, et al. Alteration of topoisomerase II-alpha gene in human breast cancer: association with responsiveness to anthracycline-based chemotherapy J Clin Oncol. 2011;29:859-67.

12. Chang JC, Wooten EC, Tsimelzon A, Hilsenbeck SG, Gutierrez MC, Elledge R, et al. Gene expression profiling for the prediction of therapeutic response to docetaxel in patients with breast cancer. Lancet. 2003;362:362-9.

13. von Minckwitz G, Untch M, Blohmer JU, Costa SD, Eidtmann H, Fasching PA, et al. Definition and impact of pathologic complete response on prognosis after neoadjuvant chemotherapy in various intrinsic breast cancer subtypes. J Clin Oncol. 2012;30:1796-804

14. Woodhams R, Matsunaga K, Iwabuchi K, Kan S, Hata H, Kuranami M, et al. Diffusion-weighted imaging of malignant breast tumors: the usefulness of apparent diffusion coefficient (ADC) value and ADC map for the detection of malignant breast tumors and evaluation of cancer extension. J Comput Assist Tomogr. 2005;29:644-9.

15. Woodhams R, Kakita S, Hata H, Iwabuchi K, Kuranami M, Gautam S, et al. Identification of residual breast carcinoma following neoadjuvant chemotherapy: diffusion-weighted imaging-comparison with contrast-enhanced MR imaging and pathologic findings. Radiology. 2010;254:357-66

16. Wu L-M, Hu J-N, Gu H-Y, Hua J, Chen J, Xu J-R. Can diffusion-weighted MR imaging and contrast-enhanced MR imaging precisely evaluate and predict pathological response to neoadjuvant chemotherapy in patients with breast cancer? Breast Cancer Res Treat. 2012;135:17-28.

17. Tozaki M, Sakamoto M, Oyama Y, Maruyama K, Fukuma E. Predicting pathological response to neoadjuvant chemotherapy in breast cancer with quantitative $1 \mathrm{H}$ MR spectroscopy using the external standard method. J Magn Reson Imaging. 2010;31:895-902.

18. Murata Y, Hamada N, Kubota K, Miyatake K, Tadokoro M, Kataoka Y, et al Choline by magnetic spectroscopy and dynamic contrast enhancement curve by magnetic resonance imaging in neoadjuvant chemotherapy for invasive breast cancer. Mol Med Rep. 2009;2:39-43.

19. Ah-See ML, Makris A, Taylor NJ, Harrison M, Richman PI, Burcombe RJ, et al. Early changes in functional dynamic magnetic resonance imaging predict for pathologic response to neoadjuvant chemotherapy in primary breast cancer. Clin Cancer Res. 2008;14:6580-9.

20. Martincich L, Montemurro F, De Rosa G, Marra V, Ponzone R, Cirillo S, et al, Monitoring response to primary chemotherapy in breast cancer using dynamic contrast-enhanced magnetic resonance imaging. Breast Cancer Res Treat. 2004;83:67-76.

21. Li SP, Makris A, Beresford MJ, Taylor NJ, Ah-See ML, Stirling JJ, et al. Use of dynamic contrast enhanced MR imaging to predict survival in patients with primary breast cancer undergoing neoadjuvant chemotherapy. Radiology. 2011;260:68-78.

22. Loo CE, Teertstra HJ, Rodenhuis S, van de Vijver MJ, Hannemann J, Muller $\mathrm{SH}$, et al. Dynamic contrast-enhanced MRI for prediction of breast cancer response to neoadjuvant chemotherapy: initial results. AJR Am J Roentgenol. 2008;191:1331-8.

23. de Bazelaire C, Calmon R, Thomassin I, Brunon C, Hamy AS, Fournier L, et al. Accuracy of perfusion MRI with high spatial but low temporal resolution to assess invasive breast cancer response to neoadjuvant chemotherapy: a retrospective study. BMC Cancer. 2011:11:361.

24. Uematsu T, Kasami M, Yuen S. Neoadjuvant chemotherapy for breast cancer: correlation between the baseline MR imaging findings and responses to therapy. Eur Radiol. 2010;20:2315-22.
25. Pickles MD, Manton DJ, Lowry M, Turnbull LW. Prognostic value of pre-treatment DCE-MRI parameters in predicting disease free and overall survival for breast cancer patients undergoing neoadjuvant chemotherapy. Eur J Radiol. 2009;71:498-505.

26. Craciunescu OI, Blackwell KL, Jones EL, Macfall JR, Yu D, Vujaskovic Z, et al. DCE-MRI parameters have potential to predict response of locally advanced breast cancer patients to neoadjuvant chemotherapy and hyperthermia: a pilot study. Int J Hyperthermia. 2009;25:405-15.

27. Bhooshan N, Giger ML, Jansen SA, Li H, Lan L, Newstead GM. Cancerous breast lesions on dynamic contrast-enhanced MR images: computerized characterization for image-based prognostic markers. Radiology. 2010:254:680-90.

28. Holli K, Lääperi AL, Harrison L, Luukkaala T, Toivonen T, Ryymin P, et al. Characterization of breast cancer types by texture analysis of magnetic resonance images. Acad Radiol. 2010;17:135-41.

29. Haralick RM, Dinstein I, Shanmugan K. Textural features for image classification. IEEE Trans Syst Man Cybern. 1973;SMC-3:610-21.

30. Zhang Y, Moore GR, Laule C, Bjarnason TA, Kozlowski P, Traboulsee A, et al. Pathological correlates of magnetic resonance imaging texture heterogeneity in multiple sclerosis. Ann Neurol. 2013;74:91-9.

31. Hajek M, Dezortova M, Materka A, Lerski R. Texture analysis for magnetic resonance imaging. Czech Republic: Med4 publishing; 2006. ISBN: 978-80-903660-0-8

32. Gibbs $P$, Turnbull LW. Textural analysis of contrast-enhanced MR images of the breast. Magn Reson Med. 2003;50:92-8.

33. Ahmed A, Gibbs P, Pickles M, Turnbull L. Texture analysis in assessment and prediction of chemotherapy response in breast cancer. J Magn Reson Imaging. 2013;38:89-101.

34. Nie K, Chen J-H, Yu HJ, Chu Y, Mehta RS, Nalcioglu O, Su M-Y. Quantitative analysis of MRI tumor characteristics for neoadjuvant chemotherapy response prediction in breast cancer to the first-line doxorubicincyclophosphamide regimen and the AC followed by Taxane Regimen. In Proceedings of the 15th International Society for Magnetic Resonance in Medicine, abstract 558. Berlin: Publisher International Society for Magnetic Resonance in Medicine (ISMRM); 2007.

35. Sahoo S, Lester SC. Pathology of breast carcinomas after neoadjuvant chemotherapy: an overview with recommendations on specimen processing and reporting. Arch Pathol Lab Med. 2009;133:633-42.

36. Mudduwa L. Pathological parameters predicting HER-2/neu status of breast carcinoma. J Diagn Pathol. 2006;5:13-8.

37. American College of Radiology. Breast imaging reporting and data system (BI-RADS). 4th ed. Reston: American College of Radiology; 2003.

38. Liney G, Gibbs P, Hayes C, Leach MO, Turnbull LW. Dynamic contrastenhanced MRI in the differentiation of breast tumours: user defined versus semi-automated region-of-interest analysis. J Magn Reson Imaging. 1999;10:945-9.

39. Likas A, Vlassis N, Verbeek JJ. The global k-means clustering algorithm. Pattern Recogn. 2003;36:451-61.

40. Tang $X$. Texture information in run-length matrices. IEEE Trans Image Process. 1998;7:1602-9.

41. Armitage $P$, Berry G, Matthews JNS. Statistical methods in medical research. 4th ed. Oxford: Blackwell Science; 2002.

42. DeLong ER, DeLong DM, Clarke-Pearson DL. Comparing the areas under two or more correlated receiver operating characteristic curves: a non parametric approach. Biometrics. 1988;44:837-45.

43. Pampel FC. Logistic regression: A primer. Thousand Oaks, California: Sage University Papers Series on Quantitative Applications in the Social Sciences: 2000. p. 7-132.

44. Baumann K. Cross-validation as the objective function for variable-selection techniques. Trends Anal Chem. 2003;22:395-406.

45. Rothman KJ. No adjustments are needed for multiple comparisons. Epidemiology. 1990;1:43-6.

46. Place AE, Jin Huh S, Polyak K. The microenvironment in breast cancer progression: biology and implications for treatment. Breast Cancer Res. 2011;13:227-38.

47. Kuhl C. The current status of breast MR imaging. Part I. Choice of technique, image interpretation, diagnostic accuracy, and transfer to clinical practice. Radiology. 2007;244:356-78.

48. Kershaw $L E$, Cheng $H-L M$. Temporal resolution and SNR requirements for accurate DCE-MRI data analysis using the AATH model. Magn Reson Med. 2010;64:1772-80 
49. Yang C, Karczmar GS, Medved M, Oto A, Zamora M, Stadler WM Reproducibility assessment of a multiple reference tissue method for quantitative dynamic contrast enhanced-MRI analysis. Magn Reson Med. 2009;61:851-9.

50. Li X, Welch EB, Chakravarthy AB, Xu L, Arlinghaus LR, Farley J, et al. Statistical comparison of dynamic contrast-enhanced MRI pharmacokinetic models in human breast cancer. Magn Reson Med. 2012;68:261-71.

51. Castellano G, Bonilha L, Li LM, Cendes F. Texture analysis of medical images. Clin Radiol. 2004;59:1061-9.

52. Ojala T, Pietikäinen M, Mäenpää T. Multiresolution gray-scale and rotation invariant texture classification with local binary patterns. IEEE Trans Pattern Anal Mach Intell. 2002;24:971-87.

53. Joshi MS, Bartakke PP, Sutaone MS. Texture representation using autoregressive models Advances in Computational Tools for Engineering Applications. In Proceedings Advances in Computational Tools for Engineering Applications, International Conference ACTEA: Beirut. 2009. 380-385: doi: 10.1109/ACTEA.2009.5227958.

54. Depeursinge A, Foncubierta-Rodríguez A, Van De Ville D, Müller H. Multiscale lung texture signature learning using the Riesz transform. In: Proceedings of the $15^{\text {th }}$ Medical Image Computing and Computer-Assisted Intervention (MICCAI), vol. 7512. Nice: Lecture Notes in Computer Science; 2012. p. $517-24$

55. Loizou CP, Murray V, Pattichis MS, Seimenis I, Pantziaris M, Pattichis CS Multiscale amplitude-Modulation frequency-modulation (AM-FM) texture analysis of multiple sclerosis in brain MRI images. IEEE Trans Info Tech Biomed. 2011;15:119-28.

56. Drabycz S, Mitchell JR. Texture quantification of medical images using a novel complex space-frequency transform. Int J CARS. 2008:3:465-75.

57. Mallat SG. A theory for multiresolution signal decomposition: the wavelet representation. IEEE Trans Pattern Anal Mach Intell. 1989;11:674-93.

58. Arlot S, Celisse A. A survey of cross-validation procedures for model selection. Stat Surveys. 2010;4:40-79.

59. Richard R, Thomassin I, Chapellier M, Scemama A, de Cremoux P, Varna M, et al. Diffusion-weighted MRI in pretreatment prediction of response to neoadjuvant chemotherapy in patients with breast cancer. Eur Radiol. 2013;23:2420-31

60. Juntu J, Sijbers J, Van Dyck D. Classification of soft tissue tumors in MR images using kernel PCA and regularized least square classifier. In: Proceedings of the $4^{\text {th }}$ conference IASTED international conference. Innsbruck: Signal Processing, Pattern Recognition, and Applications; 2007. ISBN 978-0-88986-646-1.

61. Cocquyt VF, Blondeel PN, Depypere HT, Praet MM, Schelfhout VR, Silva OE, et al. Different responses to preoperative chemotherapy for invasive lobular and invasive ductal breast carcinoma. Eur J Surg Oncol. 2003:29:361-7.

62. Tubiana-Hulin M, Stevens D, Lasry S, Guinebretière JM, Bouita L, Cohen-Solal $C$, et al. Response to neoadjuvant chemotherapy in lobular and ductal breast carcinomas: a retrospective study on 860 patients from one institution. Ann Oncol. 2006:17:1228-33.

\section{Submit your next manuscript to BioMed Central and take full advantage of:}

- Convenient online submission

- Thorough peer review

- No space constraints or color figure charges

- Immediate publication on acceptance

- Inclusion in PubMed, CAS, Scopus and Google Scholar

- Research which is freely available for redistribution

Submit your manuscript at www.biomedcentral.com/submit 